\title{
Early results of three and four part proximal humerus fractures treated with PHILOS (Proximal Humerus Interlocking System) plate
}

\begin{abstract}
Achieving stable fixation has been a challenge in proximal humerus fractures. PHILOS (Proximal Humerus internal locking system) is the latest generation of locking compression plate system for such fractures. The aim of the current study was to assess the clinical and functional outcome of proximal humeral fractures (3-part and 4-part) treated with the PHILOS plate. This was a retrospective review of first ten cases operated at our centre by a single surgeon. The patients were followed in out-patient clinics till fracture union. Fracture healing was assessed by clinical and radiological criteria, and shoulder function was evaluated using Constant and Murley score. Average follow-up was $23 \pm 3$ weeks. Eight $(80 \%)$ patients had fracture healing at an average of $11.6 \pm 0.8$ weeks post-operatively, and gained moderate range of motion. Two $(20 \%)$ patients had complications, i.e. one had deep infection and another had screw pull-out.
\end{abstract}

Conclusion: Open reduction and internal fixation with PHILOS plate showed acceptable functional outcome. Apart from the one case with screw-pull-out, our early results using this plate are encouraging, with no other implant-related complications and good early healing.

Keywords: Proximal humerus fractures, internal fixation, locking compression plate, PHILOS Plate
Volume I Issue 3 - 2014

\author{
Muhammad Ali, Muhammad A Wajid \\ Department of Surgery, Pakistan
}

Correspondence: Muhammad Ali, Department of Surgery, Agha Khan University, Block-I, House no -4 shahruknealam colony Multan, Karachi, Pakistan, Tel 923215470768 Emailmuhmmadalil9782002@yahoo.com

Received: September 29, 2014 | Published: November 09, 2014
Abbreviations: PHILOS, Proximal Humerus Interlocking System

\section{Introduction}

Fractures of the proximal humerus account for $4 \%$ to $5 \%$ of all fractures..$^{1-3}$ In patients over 65 years of age, they are the second most common upper-extremity fracture and the third most common fracture after hip fractures and distal radial fractures. ${ }^{1,4,5}$ Although most such fractures can be managed non-operatively, operative intervention is generally recommended when any of the major fracture fragments is displaced more than one centimeter or angulated more than $45^{\circ} .1,3,6$ Open reduction and internal fixation for multi-fragmented proximal humerus fractures in adults with good quality bone are not usually a problem. ${ }^{7-9}$ However in osteoporotic bones, these injuries present a real challenge. ${ }^{7,10}$ The main issue is stability of the construct; due to poor anchorage of screws in humeral head, there is a high risk of screw pullout. ${ }^{11}$ Angular stable devices such as the Proximal Humerus Interlocking System (PHILOS; Synthes, Oberdorf, Switzerland) were developed by the AO Foundation (Figure 1) provide better anchorage and superior stability. ${ }^{5,12}$ In the Aga Khan University, PHILOS was introduced into clinical practice in April 2007. In this study we present our early experience of the use of this system for 3-part and 4-part fractures of the proximal humerus.

\section{Materials and methods}

Patients with three and four part fractures of the proximal humerus who were treated surgically between April and November 2012 using PHILOS were included in the study. Ten such cases, all presenting after acute injuries and operated by a single surgeon, were identified through the hospital medical record system and chart review. A data collection form was used to record information regarding patient demography, mechanism of injury, fracture classification, technical details of surgical procedure, post-operative rehabilitation protocol and follow-up assessments.

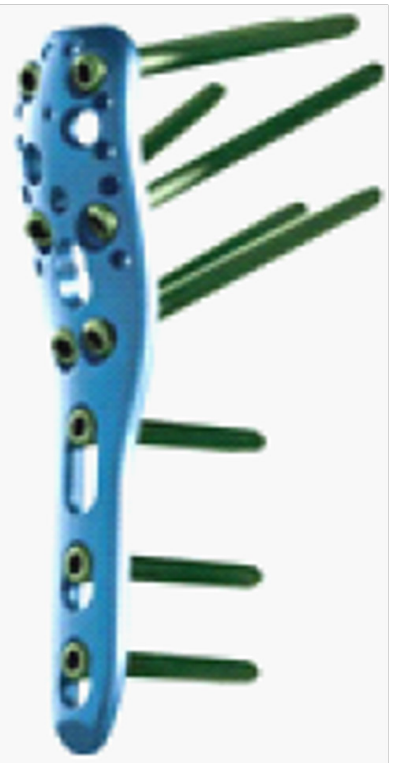

Figure I The PHILOS plate with locking head screws.

Follow-up clinical examination in the outpatient department entailed assessment of wound healing, fracture union, shoulder function and pain. Clinical fracture union was defined as fracture site becoming painless, non- tender and stable. Shoulder function was assessed using Constant and Murley scoring system. ${ }^{13}$ This score assigns points for pain, muscle strength, activities of daily life and range of motion, and is graded as poor (score $0-55$ ), moderate (56 to 70), good (71-85) and excellent (86-100). Follow-up radiographic evaluation entailed assessment of adequacy of fixation, status of the 
plate and screws, and radiological fracture union in terms of bridging of fracture gap and external callus. All fractures were classified according to AO classification. ${ }^{2}$ Surgery was performed under general anesthesia with the patient in beach chair position. An image intensifier was used for dynamic fluoroscopic monitoring of the operation. Deltopectoral surgical approach ${ }^{14,15}$ was used, taking care to protect the axillary nerve and the periosteal/muscular attachments to the bone fragments. The supraspinatus tendon was tagged with multiple \#2 braided non absorbable sutures. These tagging sutures were used to bring the tuberosity fragments to the lateral cortex of shaft fragments, thereby indirectly reducing the head fragments to the shaft. In fractures where the medial hinge was disrupted (Figure 2), attempt at restoration of the medial hinge ${ }^{16}$ was done. If head fragments were impacted on the shaft, a periosteal elevator was used to dis-impact the head and thus restore the medial portion of calcar. A minimum of four screws were placed in the proximal segment.

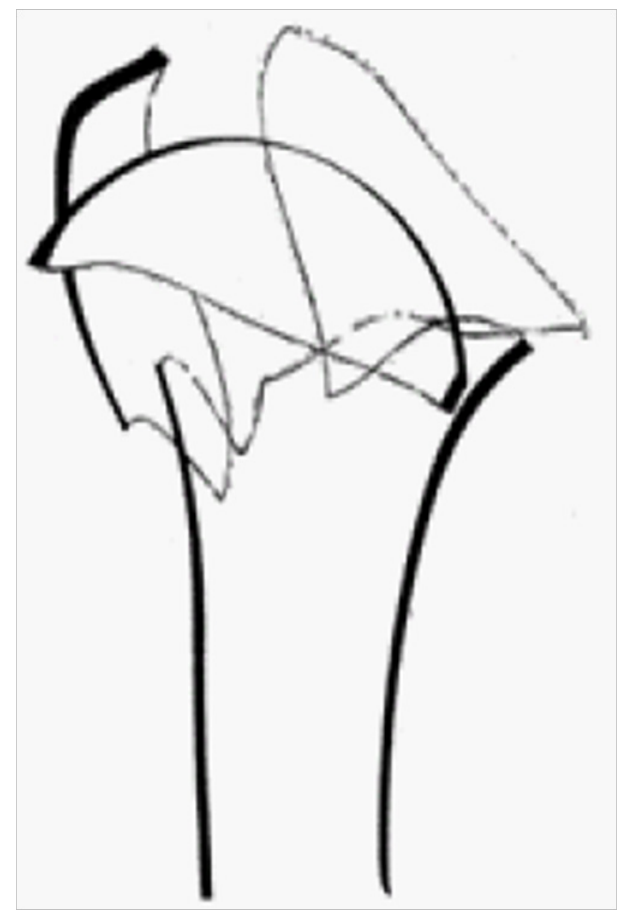

Figure 2 Four-part fracture of the proximal humerus with disrupted media hinge.

After reducing the fracture, the tagging sutures were passed through the suture hole of PHILOS. The plate was positioned directly on the middle of lateral cortex, and the proximal end was kept approximately $8 \mathrm{~mm}$ distal to the superior edge of the greater tuberosity. By using insertion guide and sleeve assembly, locked screws were placed in the humeral head. The plate was fixed to the shaft with a screw inserted in the neutral slot of the plate, and then subsequent screws were inserted in locking mode. Reproduced with permission from Sperling et al. ${ }^{17}$

\section{Results}

Of the 10 patients, 3 were male and 7 were female. Majority of the patients were elderly or middle aged. The average duration of follow up was $23 \pm 3$ (range 16-24) weeks. Eight patients had uneventful fracture union at an average of $11.6 \pm 0.8$ weeks (Table 1). The operated shoulder demonstrated mean forward flexion of $100^{\circ}$ and mean abduction of $90^{\circ}$, compared to $168^{\circ}$ and $159^{\circ}$ respectively in the contra lateral (normal) shoulder. Mean Constant score (Table 2) for the operated shoulder was 62.3 (moderate) as compared to 90.3 for the contra lateral shoulder. Radiographs demonstrated evidence of excellent healing and well positioned implants (Figure 3). None of the patients exhibited radiographic evidence of avascular necrosis at the final follow-up.

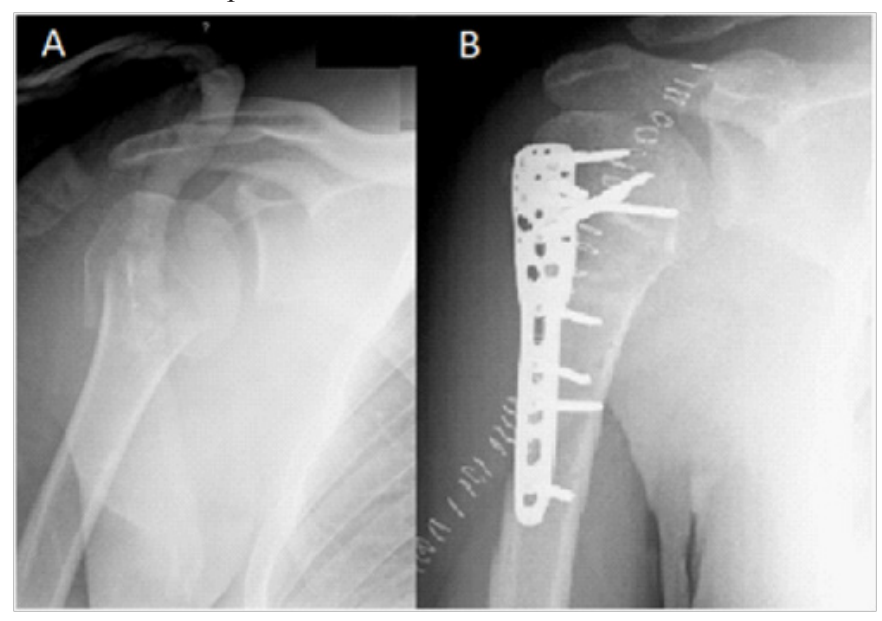

Figure 3 Antero-posterior radiograph of the shoulder showing a threepart fracture of the proximal humerus. (A) Postoperative lateral (scapular-Y) radiograph (B) showing fixation of the fracture with the PHILOS plate.

Table I Final outcome

\begin{tabular}{ll}
\hline Uneventful healing & 8 patients \\
Radiological healing time & $11.6 \pm 0.8$ weeks \\
$\begin{array}{l}\text { Complication, Requiring hemi- } \\
\text { arthroplasty }\end{array}$ & 2 patients \\
Deep infection & I Patient \\
Screw Cut-out & I Patient \\
\hline
\end{tabular}

Two patients developed complications. One had deep infection which was treated by debridement, removal of hardware and subsequent shoulder hemi-arthroplasty. The other patient had cutout of screws on follow-up at 1st week. This patient was treated by removal of hardware and hemi-arthroplasty. These patients were not included in the follow up. No patient had nerve injury, impingement or plate failure. Operating time varied according to the type of fracture. Thus, for 11-B2 fractures, mean operating time was $65 \pm 7 \mathrm{~min}$, for 11-B3 it was $88 \pm 9 \mathrm{~min}$ and for $11-\mathrm{C} 2$ it was $95 \pm 9 \mathrm{~min}$. This variation was statistically significant ( $\mathrm{p}=0.021$, ANOVA).

\section{Rehabilitation and follow-up}

We started early passive range of motion exercises followed by active exercises at 6 weeks. Resistive exercises were started at 10 weeks.

\section{Discussion}

The use of locked plating in the treatment of displaced proximal humerus fractures is becoming more wide spread. Precise knowledge of and experience with the surgical technique is required to maximize clinical outcomes, including appropriate preoperative and postoperative management. While this emerging technique has shown 
promise to date, surgeons should be aware of complications that can arise. Overall complication rates have been reported as high as $81 \%$ in the literature, depending on the definitions used, but significant variability has been present across studies. ${ }^{10-13,15}$

Table 2 Clinical and Functional Outcome. \#Increasing time according to complexity of fracture, significant ( $\mathrm{p}=0.02 \mathrm{I})$, ANOVA $*$ Trend of decreasing score according to complexity of fracture, though not significant $(p=0.206)$

\begin{tabular}{|c|c|c|c|}
\hline \multicolumn{4}{|c|}{ According to Constant and Murley score } \\
\hline Component & Max. Possible Score & $\begin{array}{l}\text { Patients' Score } \\
\text { Mean + SD (min-max) }\end{array}$ & Interpretation \\
\hline Pain & 15 & $10.6 \pm 1.8(10-15)$ & Mild \\
\hline Power & 25 & $20.6 \pm 1.8(20-25)$ & Good \\
\hline Activity Level & 10 & $4.0 \pm 0.0(4-4)$ & Full work \\
\hline Positioning & 10 & $5.8 \pm 1.7(2-8)$ & Up to neck \\
\hline Abduction and Flexion & 20 & $9.3 \pm 1.0(8-10)$ & $91^{\circ}-120^{\circ}$ \\
\hline External Rotation & 10 & $6.0 \pm 1.1(4-8)$ & Hand on top of Head, elbow forward \\
\hline Internal Rotation & 10 & $6.3 \pm 1.3(4-8)$ & Dorsum of head to waist (L3) \\
\hline Total score & 100 & $62.3 \pm 6.1(56-76)$ & \\
\hline \multicolumn{4}{|c|}{ According to Fracture type, Operating time } \\
\hline Fracture type & Number of patients & Operating time $(\mathrm{min})^{\#}$ & Constant \& Murley total score $($ mean \pm SD)* \\
\hline II-B2 & 2 & $65 \pm 7$ & $68 \pm 11$ \\
\hline II-B3 & 4 & $88 \pm 9$ & $62 \pm 2$ \\
\hline $1 \mathrm{I}-\mathrm{C} 2$ & 2 & $95 \pm 9$ & $57 \pm 1$ \\
\hline
\end{tabular}

In general, non-operative treatment of displaced three- and four-part fractures of the proximal humerus, which form 13\%-16\% of proximal humeral fractures, leads to poor outcome due to intraarticular nature of injury and inherent instability of the fragments. ${ }^{3,5}$ A careful assessment of the patient in terms of age, activity level, bone quality, fracture pattern, degree of comminution and vascular status of the fracture fragments is required before committing to the type of treatment to be used in a particular patient. ${ }^{7,8}$ Operative treatment is challenging in terms of fixation and stability of construct in comminuted fractures and osteoporotic bone. ${ }^{3,7}$ This is a fairly common scenario in elderly patients, in whom osteoporosis leads to comminuted fractures of the neck and head of humerus. Conventional plating in such fractures leads to unacceptably high incidence of screw pullout. ${ }^{5}$ From historical comparisons and biomechanical evaluation of locked internal fixator we know that pull out strength of locked head screws is better than conventional screws due to the axial and angular-stability of screws. ${ }^{11,12}$ The PHILOS, and modified cloverleaf plate with locking head screws are examples of new implants analogous to locked internal fixators. ${ }^{4,5,12}$ The combination of locking head screws with three dimensional positioning of the screws within the humeral head leads to improved stability. Fractures of the AO-type 11-A3, 11-B2, 11-B3, 11-C2 and reconstructable 11-C3 fractures have been the most frequent indications for fixation with this new system. ${ }^{2}$ Although PHILOS has superior stability compared to conventional plate in fractures in the elderly with osteoporosis, in cases with severely osteoporotic and multi-fragmented fractures, the screws may cut out of the bone despite the locking heads. ${ }^{7}$ The insertion of this new device is technically demanding; in particular the insertion of the proximal screws entails the risk of perforation of the sub-chondral bone by the screw tip. This is because of the sphericity of the humeral head and misleading assessment of the length of screw under fluoroscopy. ${ }^{14,15}$ Reconstruction of the medial buttress in the metaphyseal area of humerus is a key point in fixation of proximal humerus with proximal humerus interlocking system. ${ }^{16}$ If the medial buttress can be restored by indirect technique, this leads to a very stable fixation and allows early range of motion. If there is medial comminution or bone defect, the medial buttress can be restored by impacting the shaft in to head and fixing it in the position with proximal humerus interlocking system. ${ }^{14-16}$

Open reduction technique and internal fixation with the conventional T- plate requires exact preparation of the fracture fragments in order to seat the plates on to the bone, causing additional damage to the already compromised blood supply and leading to a high rate of avascular necrosis of the humeral head. ${ }^{3,5}$ The rate of avascular necrosis has been reduced by using minimally invasive approach, indirect reduction techniques and minimal soft tissue disruption..$^{5,18}$ Anatomically pre-shaped ${ }^{19}$ implants like the PHILOS generally don't require contouring and do not need to be seated on the bone, thus eliminating the need for muscular/periosteal disruption. Avascular necrosis may be predicted on the initial fracture radiographs using the Hertel ${ }^{18}$ radiographic criteria for perfusion of humeral head. According to this criteria, metaphyseal extension of humeral head of $<8 \mathrm{~mm}$ and disruption of medial hinge of $>2 \mathrm{~mm}$ are good predictors of ischemia. These, along with anatomical neck fracture pattern, have a $97 \%$ of positive predictive value for humeral head ischemia. Such fractures are better treated using hemi-arthroplasty instead of internal fixation. ${ }^{3,5}$

Koukakis et al. ${ }^{20}$ described their experience using the proximal humeral internal locked system (PHILOS) plate in the operative management of proximal humerus fractures. Over a 3-year period, 20 patients with a mean age of 62 years had their fractures treated with this locked plate construct. The authors reported a $100 \%$ rate of fracture union. These results are comparable to our study. Apart from 
the two cases one with screw pull-out and other with deep infection, our results showed that fracture union was achieved in all cases, and none developed avascular necrosis. Moreover, shoulder pain was mild and function was moderate at the final follow-up. The main reason for the "moderate" instead of "good" outcome were the low activity level and inability to achieve abduction and flexion beyond $120^{\circ}$. These could be attributed to the sedentary lifestyle of the patient population who were mostly elderly/middle aged people with low functional demands. In appropriately selected cases, PHILOS appears to provide adequate stability of the fracture fragments and acceptable functional outcome. The limitation in our study was absence of control group of patients treated conservatively or with an alternative device. Secondly, absolute confirmation of screw placement is not possible with biplanar fluoroscopy.

\section{Conclusion}

The use of locked plating in the treatment of displaced proximal humerus fractures is becoming more prevalent. The primary goal of surgery should be to create a construct stable enough to allow early ROM of the shoulder. Precise knowledge of and experience with the surgical technique is required to maximize clinical outcomes, including appropriate preoperative and postoperative management. While this emerging technique has shown promise to date, surgeons should be aware of complications that can arise. Our early results on the use of this plate are encouraging, and it appears that use of PHILOS in three and four part humerus fractures, particularly in osteopenic bone, provides acceptable results.

\section{References}

1. Neer CS. Displaced Proximal Humeral Fractures. I Classification and Evaluation. J Bone Joint Surg Am. 1970;52(6):1077-1089.

2. Plecko M.Humerus, proximal. In: Wagner M, Frigg R. AO Manual of internal fixators. Concepts and cases using LCP and LISS. Davos. AO, 2006; pp. 224-229.

3. Nho SJ, Brophy RH, Barker JU, et al. Management of Proximal Humeral Fractures Based on Current Literature. J Bone Joint Surg Am. 2007;89(Suppl 3):44-58.

4. Shahid R, Mushtaq A, Northover J, et al. Outcome of proximal humerus fractures treated by PHILOS plate internal fixation. Experience of a district general hospital. Acta Orthop Belg. 2008;74(5):602-608.

5. Friess DM, Attia A. Locking plate fixation for proximal humerus fractures: a comparison with other fixation techniques. Orthopedics. 2008; 31(12).

6. Handschin AE, Cardell M, Contaldo C, et al. Functional results of angular-stable plate fixation in displaced proximal humeral fractures. Injury. 2008;39(3):306-313
7. Solberg BD, Moon CN, Franco DP, et al. Locked plating of 3- and 4-part proximal humerus fractures in older patients: the effect of initial fracture pattern on outcome. J Orthop Trauma. 2009;23(2):113-119.

8. Helwig P, Bahrs C, Epple B, et al. Does fixed-angle plate osteosynthesis solve the problems of a fractured proximal humerus? A prospective series of 87 patients. Acta Orthop. 2009;80(1):92-96.

9. Thanasas C, Kontakis G, Angoules A, et al. Treatment of proximal humerus fractures with locking plates: A systematic review. $J$ Shoulder Elbow Surg. 2009;18(6):837-844.

10. Ricchett ET, Warrender WJ, Abboud JA. Use of locking plates in the treatment of proximal humerus fractures. J Shoulder Elbow Surg. 2010;19(2Suppl):66-75

11. O'Toole RV, Andersen RC, Vesnovsky O, et al. Are locking screws advantageous with plate fixation of humeral shaft fractures? a biomechanical analysis of synthetic and cadaveric bone. J Orthop Trauma. 2008;22(10):709-715

12. Thalhammer G, Platzer P, Oberleitner G, et al. Angular stable fixation of proximal humeral fractures. J Trauma. 2009;66(1): 204-210.

13. Constant CR, Murley AH. A clinical method of functional assessment of the shoulder. Clin Orthop Relat Res. 1987;(214):160-164.

14. Hepp P, Theopold J, Voigt C, et al. The surgical approach for locking plate osteosynthesis of displaced proximal humeral fractures influences the functional outcome. J Shoulder Elbow Surg. 2008;17(1):21-28.

15. Konrad G, Bayer J, Hepp P, et al. Open reduction and internal fixation of proximal humeral fractures with use of the locking proximal humerus plate: Surgical technique. J Bone Joint Surg Am. 2010;92(Suppl 1):85-95.

16. Gardner MJ, Weil Y, Barker JU, et al. The importance of medial support in locked plating of proximal humerus fractures. J Orthop Trauma. 2007;21(3):185-191.

17. Sperling JW, Cuomo F, Hill JD, et al. The difficult proximal humerus fracture: tips and techniques to avoid complications and improve results. Instr Course Lect. 2007;56:45-57.

18. Greiner S, Kaab MJ, Haas NP, et al. Humeral head necrosis rate at midterm follow-up after open reduction and angular stable plate fixation for proximal humeral fractures. Injury. 2009;40(2):186-191.

19. Ricchetti ET, DeMola PM, Roman D, et al. The use of precontoured humeral locking plates in the management of displaced proximal humerus fracture. J Am Acad Orthop Surg. 2009;17(9):582-590.

20. Koukakis A, Apostolou CD, Taneja T, et al. Fixation of proximal humerus fractures using the PHILOS plate: early experience. Clin Orthop Relat Res. 2006;442:115-120. 